\title{
T-Cell Subsets as Potential Biomarkers for Hepatobiliary Cancers and Selection of Immunotherapy Regimens as a Treatment Strategy
}

\author{
Chandan Kumar-Sinha, PhD, ${ }^{1,2}$ and Vaibhav Sahai, MBBS, MS $\mathrm{M}^{3,4}$
}

\begin{abstract}
Patients with advanced hepatocellular or biliary cancers have a dismal prognosis with limited efficacy from standard systemic therapies. The benefit of precision medicine has so far been limited to a subset of biliary cancers, including FGFR rearrangements; hotspot mutations in IDH1/2, BRAF, and BRCA1/2; and other rare alterations. In contrast, hepatocellular carcinoma, an inflammation-driven cancer with an immune-infiltrated microenvironment, provides a promising opportunity for immunotherapy, compared with the highly desmoplastic immune desert or excluded stromal microenvironment in biliary cancers. The immune contexture in hepatobiliary cancers is mostly immunosuppressive, protumorigenic, and exhausted, which together with low tumor mutation burden and decreased neoantigens provides challenges for immunotherapy. A better understanding of the spatiotemporal profile of $\mathrm{T}$ cells within the tumor microenvironment and the dynamic interplay of immune modulators in the context of standard or experimental therapies is crucial to define additional markers of response and design evidence-based combinatorial regimens. This review considers recent literature in this area and highlights promising leads and emerging trends.
\end{abstract}

J Natl Compr Canc Netw 2022;20(2):203-214 doi: $10.6004 /$ jnccn.2021.7097

${ }^{1}$ Michigan Center for Translational Pathology, ${ }^{2}$ Department of Pathology,

${ }^{3}$ Division of Hematology and Oncology, Department of Internal Medicine, and

${ }^{4}$ Rogel Cancer Center, University of Michigan, Ann Arbor, Michigan.
Hepatobiliary cancers (HBTCs) include hepatocellular carcinoma (HCC) and biliary tract cancers (BTCs), which further include gallbladder cancer (GBC) and cholangiocarcinoma (CCA; intrahepatic [iCCA] and extrahepatic). Patients with advanced HBTCs have limited options for treatment. High-throughput genomic studies have delineated landscapes of somatic aberrations driving HBTCs. ${ }^{1,2}$ The subsets harboring therapeutically actionable targets in HCC include receptor tyrosine kinases (RTKs), such as MET and CCND1 amplification in HCC. ${ }^{3,4}$ Similar actionable subsets in BTC include FGFR2 rearrangements, IDH1/2 and $B R A F$ mutations in iCCAs, ${ }^{5-8}$ and $R A S / R T K$ gene aberrations, such as HER2 amplification in extrahepatic CCA and GBC. ${ }^{8-10}$ Unfortunately, apart from modest success with multi-tyrosine kinase inhibitors ${ }^{4}$ and dual anti-PD-1 and vascular endothelial growth factor (VEGF) antibody therapy in HCC, and with panFGFR inhibitors, ${ }^{11-13}$ IDH1 inhibitors, dual BRAF and MEK inhibitors, and anecdotes of benefit from targeted precision therapies ${ }^{14-16}$ in BTC, most patients with HBTC remain in urgent need of breakthroughs in treatment options.

The recent success of immune checkpoint inhibitor (ICI) therapy with the anti-PD-L1 antibody atezolizumab, in combination with the VEGF antibody bevacizumab, has led this combination to be used as the first-line therapy in patients with advanced HCC. In addition, anti-PD-1 therapy has tumor-agnostic FDA approval for patients with uncommon molecular subsets of high tumor mutation burden (TMB), high microsatellite instability (MSI-H), or deficient mismatch repair proteins (dMMR). ${ }^{17-21}$ Furthermore, anti-PD-1 nivolumab and pembrolizumab monotherapy and the combination of nivolumab with the anti-CTLA-4 antibody ipilimumab are approved as the second-line treatment of HCC after sorafenib, based on early- and late-phase trial results. ${ }^{22-24}$ In contrast, a similar benefit has not been realized in BTCs, wherein only a small subset of unselected patients have shown durable response to ICIs in clinical trials, 
and the molecular subset of high TMB and MSI-H/ dMMR is no higher than $5 \%$ of the patient population. This finding has motivated a more comprehensive characterization of the tumor immune microenvironment and systemic immune factors that together determine the response to immunotherapy. ${ }^{25}$

This review presents a brief overview of recent literature highlighting the tumor microenvironment (TME) immune milieu in HBTCs, with specific emphasis on T-cell subsets and immunotherapeutic strategies to overcome barriers to treatment response.

\section{TME and Tumor-Infiltrating Lymphocytes}

\section{Hepatocellular Carcinoma}

The tumor-infiltrating lymphocytes (TILs) repertoire of HCC tumors represents a varied mix of both immuneactivating and immune-suppressive factors that include subtypes of T-helper cells, $\mathrm{CD}^{+}{ }^{+} \mathrm{T}$ cells, natural killer $\mathrm{T}$ cells, and regulatory $\mathrm{T}$ cells (Tregs), ${ }^{26-28}$ with no significant difference noted across different etiologies of cirrhosis, such as hepatitis B or C or alcoholic or nonalcoholic steatohepatitis, or from disease stage as shown in one study. ${ }^{29}$ However, a tumor-promoting milieu composed of exhausted $\mathrm{CD} 8^{+} \mathrm{PD}-1^{+} \mathrm{CXCR}^{+}{ }^{+}$cells with impaired tumor immune surveillance was found enriched in nonviral, nonalcoholic steatohepatitis-affected livers compared with other etiologies. ${ }^{30}$ Consistent with this finding, a meta-analysis of patients with advanced HCC treated with PD-L1/PD-1 inhibitors showed inferior survival among those with nonviral HCC. A comprehensive transcriptomic analysis of the HCC TME comparing data from The Cancer Genome Atlas (TCGA; HCC) and the Genotype-Tissue Expression project (GTEx; normal liver) defined an 89-gene signature with increased expression of T-cell markers, cytolytic activity, and T-cell costimulation, associated with a favorable prognosis. ${ }^{29}$ Elucidation of the complex and dynamic interplay of cancer cells, stromal fibroblasts, and tumor-infiltrated immune cells involving hepatic stellate cells, cancer-associated fibroblasts, neutrophil extracellular traps, and myeloidderived suppressor cells, among others, comprising the HCC tumor immune milieu is an area of intensive investigation. ${ }^{31-33}$ Another notable, emerging facet of the HCC immune milieu involves the potential role of the microbiome, highlighted by Ma et al, ${ }^{34}$ who showed that the bowel and bile microbiome was associated with the accumulation of $\mathrm{CXCR}^{+}$hepatic natural killer $\mathrm{T}$ cells and an altered tumor immune response.

\section{Biliary Tract Cancer}

In contrast to HCC, the BTC microenvironment has been noted for an immune-excluding desmoplastic-rich stroma, cancer-associated fibroblasts, endothelial cells, and mostly immune cold or suppressive immune subsets (Figure 1). ${ }^{35,36}$ Tumor-associated macrophages (TAMs) represent the predominant population of infiltrating immune cells in CCA tumors, and elevated levels are associated with poor prognosis, reduced survival, and metastasis. ${ }^{37}$ Job et $\mathrm{al}^{38}$ recently defined 4 subtypes of iCCA TMEs based on gene expression profiling of iCCA tumors $(n=566)$ and microdissected epithelial/stromal compartments: (1) an immune desert (46\%-48\%), marked by a lack of $\mathrm{CD} 8^{+} \mathrm{T}$ cells, major histocompatibility complex (MHC) class I cells, and myeloid cells; (2) mesenchymal (22\%-28\%), with an immune-excluded niche rich in fibrous stroma, vascular factors, and chemokines; (3) myeloid (13\%-19\%), marked by M2 macrophages and $\mathrm{CD}^{+} / \mathrm{CD}^{+} \mathrm{T}$ cells; and (4) inflamed/ immunogenic $(9 \%-13 \%)$, rich in both immune-stimulating and immune-suppressive features (Figure 1). The intratumoral immune tolerance is also thought to be a result of the "exhaustion" of the proinflammatory T cells and the accumulation of Tregs, myeloid-derived suppressor cells, and TAMs that permit tumor progression and metastasis.

$\mathrm{CD}^{+} \mathrm{T}$ cells

$\mathrm{CD}^{+}$cells are classified into T-helper cells (Th1, Th2, Th9, Th17) and Tregs based on cytokine profiles. Broadly, T-helper cells are responsible for modulating cytotoxic T-cell activity. T-helper cell subsets Thl and Th17 promote cell-mediated immune responses and inflammation by cytokine production (such as IFN- $\gamma$, IL-2, IL-la, IL-1b, and IL-17), whereas Th2 cells are involved in humoral responses via B lymphocytes and may promote tumor tolerance. Tregs mediate immunosuppression via the release of cytokines, such as transforming growth factor $\beta$ (TGF- $\beta$ ), IL-10, and IL-35. Other protumorigenic $\mathrm{CD}^{+}$cells identified in HCC include exhausted $\mathrm{CD}^{+}{ }^{+} \mathrm{T}$ cells coexpressing PD-1 and BTLA (B and T lymphocyteassociated), ${ }^{39}$ Thl cells that produce IL-2 and IFN- $\gamma$, and exhausted or low follicular T-helper cells, mucosal-associated invariant $\mathrm{T}$ cells, ${ }^{40}$ and cytokine-producing Th17, Th2, and Th9 cells. ${ }^{41,42}$

A differential enrichment of T-cell subsets has been noted in BTC in a spatially distinct manner with a higher density of Treg $\left(\right.$ Foxp $^{+}$) cells in the tumor center, and cancer cells displaying an epithelial-to-mesenchymal transition phenotype surrounded by IL-17-producing Th17 cells composing the tumor front, associated with cancer progression. ${ }^{43}$ These distinct T-cell subtypes have been mechanistically linked to the expression of cytokine IL- 6 and TGF- $\beta 1$ based on in vitro coculture experiments. Along similar lines, Ikemoto et $\mathrm{al}^{44}$ described increased levels of 2 populations of Tregs-Type 1 (Trl; CD4 ${ }^{+} \mathrm{CD}_{4}$ 9b ${ }^{+}$LAG3 $^{+}$) and Foxp3 ${ }^{+}-$ in both peripheral and intratumoral settings in recurrent HCC and BTC that were associated with low survival. 


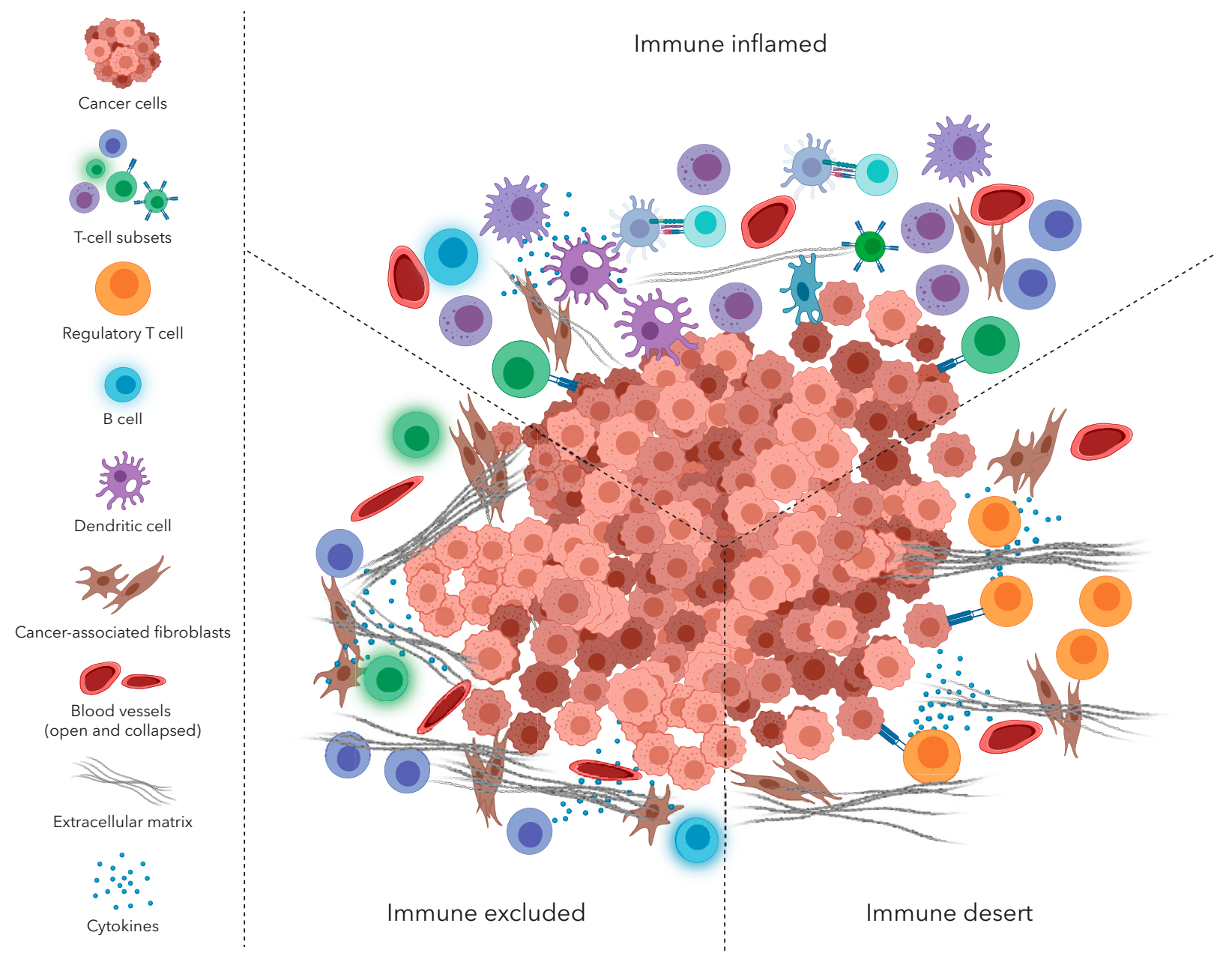

Figure 1. Tumor immune microenvironment.

$\mathrm{CD}^{+} \mathrm{T}$ cells

$\mathrm{CD}^{+}$cells are cytotoxic $\mathrm{T}$ cells and are classified into $\mathrm{Tc} 1$ and Tc2 subtypes. High tumor infiltration of $\mathrm{CD}^{+} \mathrm{T}$ cells in HCC is generally associated with better survival and lower recurrence. ${ }^{45}$ The $\mathrm{CD}^{+}{ }^{+} \mathrm{T}$-cell population in HCC tends to be PD-1-high ${ }^{38}$; however, PD-1 expression on $\mathrm{T}$ cells has a dual role and may reflect both activation and dysfunction. Indeed, HCCs showing higher levels of $\mathrm{CD}^{+} \mathrm{T}$ cells overexpressing coinhibitory receptors such as CTLA-4, PD-1, LAG3, and TIM-3 show an exhausted phenotype and are associated with poor clinical outcome. ${ }^{46}$ The exhaustion of $\mathrm{CD} 4^{+} \mathrm{Th} 1$ and $\mathrm{CD} 8^{+}$cytotoxic $\mathrm{T}$ cells within the tumor milieu is multifactorial; however, the PD-1/PD-L1 immune checkpoint is a key driver of immune exhaustion in HCC, and perhaps in BTC as well.

In a meta-analysis of data from 2,339 patients with BTC across 12 studies, high levels of $\mathrm{CD} 66 \mathrm{~b}^{+}$neutrophils, a high neutrophil-to-lymphocyte ratio, and presence of intratumoral $\mathrm{IL}-17^{+}$cells and $\mathrm{PD}-1^{+} / \mathrm{CD} 8^{+}$TILs were associated with poorer overall survival (OS), whereas high levels of $\mathrm{CD}^{+}$and $\mathrm{CD}^{+}{ }^{+} \mathrm{T}$ cells, MHC class I-presenting cells, and NKG2D cells were associated with better OS. ${ }^{47} \mathrm{~A}$ high neutrophil-to-lymphocyte ratio in the preoperative peripheral blood of patients with BTC was correlated with lower tumor-infiltrating $\mathrm{CD} 8\left({ }^{+}\right) \mathrm{T}$ cells and poorer OS and recurrence-free survival, suggesting a potential prognostic utility. $^{48}$ In a cohort of patients with iCCA $(n=54)$, PD-L1 expression was seen in the TAMs and on tumor cells within the tumor front in 34 and 39 patients, respectively. Interestingly, PD-L1 expression on tumor cells within the tumor front but not $\mathrm{TAMs}^{+}$was associated with a $60 \%$ decreased survival and was also negatively correlated with lymph node and intrahepatic metastasis. ${ }^{49}$ Similarly, iCCAs with $\mathrm{CD}^{+}{ }^{\mathrm{PD}}-1^{\text {high }} \mathrm{T}$ cells in conjunction with $\mathrm{CD} 8^{+}$ PD-L1 $\left.{ }^{+}\right)$TAMs were significantly correlated with advanced 
TNM stage and worse postoperative survival. ${ }^{50}$ The spatial context of cancer and immune cells is also underscored in extrahepatic CCA, in which $32.3 \%$ of tumors with adjacent normal cells positive for PD-L1 and PD-1 were associated with a higher density of $\mathrm{CD}^{+}{ }^{+} \mathrm{T}$ cells and improved survival. ${ }^{51}$ The role of the immune milieu in the adjacent normal tissue has also been identified via transcriptomic profiling in another small cohort $(n=22)$ of matched BTC tumor and adjacent normal tissue. Specifically, CTLA-4 expression in adjacent normal tissue was correlated with Treg cells and inversely correlated with recurrence-free survival. ${ }^{52}$ Analysis of $\mathrm{CD}^{+}{ }^{+}$T-cell density and PD-L1 expression in GBCs $(n=66)$ identified $54 \%$ of patients as having PD-L1 $\left(^{+}\right)$(18\% and $36 \%$ in tumor cells and peritumoral immune stroma, respectively). High $\mathrm{CD}^{+} \mathrm{T}$-cell density was observed in these PD-Ll( $\left(^{+}\right)$GBCs and was associated with improved OS and progression-free survival (PFS). ${ }^{53}$

Interestingly, the density of PD- $1\left(^{+}\right) \mathrm{CD}^{+} \mathrm{T}$ cells in BTCs $(n=37)$ may decrease during progression from early- to late-stage disease. ${ }^{54}$ This finding corroborated in a mouse model, wherein PD-1 expression by tumor-infiltrating $\mathrm{CD}^{+} \mathrm{T}$ cells was higher in smaller tumors and decreased with tumor growth. ${ }^{54}$ Consequently, large tumors were less responsive to PD-1 blockade, whereas small tumors containing higher numbers of PD $-1^{+} \mathrm{CD} 8^{+}$ $\mathrm{T}$ cells were sensitive. Another noteworthy observation in patients with iCCA with potential therapeutic implications is the elevated level of immune inhibitory checkpoint molecule HHLA2 in 50\% of PD-L1-negative tumors, which was associated in one study with a sparser population of $\mathrm{CD}^{+} \mathrm{T}$ cells and a higher ratio of Treg/ $\mathrm{CD}^{+}$cells, thus supporting an immune-suppressed TME. ${ }^{55}$ In this cohort, HHLA2 expression, but not PDL1, was significantly associated with worse OS, suggesting that anti-PD-1/CTLA-4 therapies may not be useful in this cohort and may warrant the development of an HHLA2 immune checkpoint blockade..$^{55}$ In iCCA, PD-1/PD-L1 expression has been significantly associated with hepatitis B virus infection and lymph node invasion in a cohort of 320 patients with iCCA; patients with advanced iCCA with hepatitis B virus infection and fewer PD- $1\left(^{+}\right) \mathrm{T}$ cells tended to have good response to anti-PD- 1 therapy. ${ }^{56}$

The complexity of this immune contexture, including but not limited to poor infiltration and spatial context of TILs, the immunosuppressive role of other immune cells such as TAMs and myeloid-derived suppressor cells, dysfunctional antigen presentation, tumor-metabolicimmune crosstalk, cytotoxic T-cell exhaustion, and multiple immune checkpoints, explains the lack of a clear prognostic and predictive association with TILs or an individual gene expression, such as PD-L1, in response to PD-1/PD-L1 ICI therapy.

\section{Targeting the Immune Microenvironment}

Effective immunotherapy for HBTCs is currently limited because of the complex nature of the immune microenvironment along with a high proportion of immune-suppressed tumors characterized by rich stroma with an immune-excluded or "cold" microenvironment, especially in BTCs, and a relatively low frequency of patients with MSI-H/dMMR or high TMB. Immunotherapy clinical trials involving patients with HBTC have been reviewed recently ${ }^{57}$; this section highlights key insights and emerging trends.

\section{PD-1/PD-L1}

High PD-1 expression in TILs and correlation between an exhausted phenotype and impaired effector function have been observed in HBTC, as noted earlier. In patients with advanced BTC after progression on at least one line of chemotherapy, monotherapy using anti-PD-1 led to an overall response rate (ORR) of only $5 \%$ to $11 \% .{ }^{58,59}$ In contrast, anti-PD-1 or anti-PD-L1 monotherapy has shown robust and often durable objective responses in a subset of $15 \%$ to $20 \%$ of patients with previously treated advanced HCC, which led to accelerated FDA approvals and inclusion in NCCN Clinical Practice Guidelines in Oncology (NCCN Guidelines) for Hepatobiliary Cancers. ${ }^{60-62}$ Interestingly, baseline PD-L1 expression in tumor cells did not have a significant impact on ORR.$^{60}$ However, subsequent phase III trials in patients with treatmentnaïve advanced HCC have failed to show improvement in survival using first- or second-line treatment compared with sorafenib or placebo, respectively. ${ }^{23,63}$

The phase II KEYNOTE-158 trial tested pembrolizumab in patients with advanced, previously treated, unresectable or metastatic TMB-high solid tumors, including in a subset of BTCs $(n=22)$, and noted an ORR of $34.3 \%$ and a median OS of 23.5 months, ${ }^{64}$ which led to a tissueagnostic FDA approval. Unfortunately, $>95 \%$ of patients with BTC are still in need of predictive markers because monotherapy using nivolumab, ${ }^{58}$ durvalumab, ${ }^{65}$ or pembrolizumab $^{59}$ in this unselected patient population did not show promising efficacy.

\section{CTLA-4}

Given that only small subsets of patients with HBTC have shown a response to ICI monotherapy, the current focus is on combining ICI with other therapies (including alternative forms of immunotherapy) in an effort to boost antitumor immunity and alter the immune microenvironment. Because CTLA-4 expression is a marker of T-cell exhaustion and engages a different target and mechanism of action, it has been explored in HCC in combination with anti-PD-1/PD-L1 inhibitors. ${ }^{66}$ Indeed, the combination of nivolumab + ipilimumab in the CheckMate 040 phase I/II trial showed manageable safety, a durable ORR of $32 \%$, 
and a median OS of 22.8 months in patients with advanced HCC previously treated using sorafenib $(n=148)$, which led to an accelerated approval. ${ }^{24}$ Similarly, the combination of durvalumab and tremelimumab showed an ORR of $24 \%$ and a median OS of 18.7 months in a phase II trial in patients with advanced HCC. ${ }^{22}$ However, dual ICI trials in BTC have not reported consensus outcomes, with a potential benefit reported in previously treated and untreated patients. ${ }^{65,67,68}$ Additional trials involving similar combinations are underway (eg, ClinicalTrials.gov identifier: NCT03473574) (Table 1).

\section{Other Immune Checkpoints}

Additional T-cell coinhibitory molecules serving as immune checkpoints and being explored as potential biomarkers and targets for immunotherapy include LAG3, HAVCR2 or TIM3, and TIGIT or VSTM3. LAG3 is typically coexpressed with PD-1, and promising clinical response has been noted with dual targeting of LAG3 and PD-1 in multiple clinical trials involving both solid tumors and hematologic malignancies. ${ }^{69}$ In the context of HBTCs, LAG3 expression has been noted in the stroma of $\mathrm{HCC}^{70}$ and CCA. ${ }^{71}$ Ongoing clinical trials are testing LAG3 inhibitors in HCC, such as INCAGN02385 and relatlimab (ClinicalTrials.gov identifiers: NCT03538028 and NCT04658147).

\section{Chemoimmunotherapy}

Platinum-based chemotherapy has been associated with immunomodulation, including the upregulation of PDL1 expression in tumor tissues that may then be targeted by PD-1/PD-L1 inhibitors. ${ }^{72}$ A phase II trial of gemcitabine, cisplatin, and nivolumab in 30 patients in Japan with BTC who were treatment-naïve showed worse PFS and only a modest improvement in response rate and OS compared with historical control patients. A similar multicenter phase II trial of a gemcitabine/cisplatin/nivolumab combination in 32 US patients with BTC who were treatment-naïve reported no difference in median OS compared with historical control patients. ${ }^{68}$ In contrast, a gemcitabine/cisplatin/durvalumab combination \pm tremelimumab in 121 Korean patients with BTC who were treatment-naïve showed promising efficacy with durable ORR and PFS. ${ }^{73}$ The combination of gemcitabine, cisplatin, and anti-PD-1/PD-L1 is now being tested in phase III trials (ClinicalTrials.gov identifiers: NCT03875235, NCT04003636, and NCT04677504). Recently, the results of the phase III trial investigating gemcitabine, cisplatin \pm durvalumab were released, stating that the durvalumab + chemotherapy arm met the primary endpoint of OS. ${ }^{74}$

\section{Locoregional Therapy and Immunotherapy}

Locoregional therapy (LRT), including ablation, embolization, selective internal radiotherapy, and radiation, is used as initial therapy in early-stage HCCs and iCCA. Multiple trials are now evaluating the role of systemic ICIs in combination with LRTs in these early-stage HBTCs to perhaps either stimulate an early immune response to prevent recurrence or obtain an abscopal effect for improved local control. Initial data reported from a study with 42 patients with HCC treated using selective internal radiotherapy followed by nivolumab showed an ORR of $38 \%$ (including $11.9 \%$ complete response), median time to progression of 9.3 months, and median OS of 20.6 months. ${ }^{75}$ In comparison, the combination of microwave ablation with tremelimumab in 20 patients with refractory CCA showed an ORR of $12.5 \%$, a median PFS of 3.4 months, and a median OS of 6.0 months. ${ }^{76}$ Multiple other LRT-plus-ICI trials continue to study the potential local and abscopal effect of this intriguing combination in HBTCs (Tables 1 and 2).

\section{Factors Regulating Tumor-TME Interaction: Additional Avenues for Therapy}

Apart from the noted responsiveness of ICI to genomic aberrations such as MSI-H/dMMR or high TMB, ${ }^{77}$ only a few other specific associations have been identified. No specific driver mutations in BTCs have been associated with any specific immune cell infiltration, but retrospective analyses of samples from patients treated using immunotherapies can potentially be informative. ${ }^{78}$

\section{VEGF Pathway}

Multi-tyrosine kinase inhibitors, such as sorafenib, lenvatinib, cabozantinib, and regorafenib, along with ramucirumab, a VEGFR2 monoclonal antibody, target the overactive VEGF pathway and are FDA-approved as monotherapies in advanced HCC. However, antiVEGF therapies may possibly reduce immunosuppression and promote T-cell infiltration, ${ }^{79}$ providing a rationale to combine with ICI therapies. Indeed, the combination of atezolizumab and bevacizumab in patients with advanced unresectable HCC showed significant improvement in efficacy when compared with sorafenib in a phase III trial ${ }^{80}$ that led to a recent FDA approval. Similarly, in BTC, a lenvatinib/pembrolizumab combination, showed modest efficacy with an ORR of $25 \%$ and a median OS of 11 months in a cohort of 32 previously treated patients with BTC. ${ }^{81}$ However, ramucirumab with pembrolizumab showed a lack of efficacy, with an ORR of $4 \%$, a median PFS of 1.6 months, and an OS of 6.4 months in a similar patient population with BTC $(n=26){ }^{82}$ Multiple other trials using this therapeutic combination are either ongoing or being planned, including cabozantinib and pembrolizumab (ClinicalTrials.gov identifier: NCT04442581) in HCC and a gemcitabine/cisplatin/atezolizumab/bevacizumab combination in BTC (NCT04677504). 


\begin{tabular}{|c|c|c|c|c|c|c|}
\hline Combination & Trial Characteristics & Targets & Agents & $\begin{array}{l}\text { ClinicalTrials.gov } \\
\text { Identifier }\end{array}$ & Phase & $\mathbf{N}$ \\
\hline \multicolumn{7}{|l|}{ PD-1/PD-L1 \pm other 10} \\
\hline Monotherapy & Multicancer; advanced stage & PD-1 & Pembrolizumab & NCT02628067 & II & 1,595 \\
\hline \multirow[t]{5}{*}{ PD-1/PD-L1 + other } & Advanced stage & PD-1 + TIM-3 & TSR-022 and TSR-042 & NCT03680508 & "I & 42 \\
\hline & Multicancer; advanced stage & PD-1 + CTLA-4 & Ipilimumab, nivolumab & NCT02834013 & ॥ & 818 \\
\hline & Multicancer; advanced stage & PD-1 + GM-CSF & Pembrolizumab, sargramostim & NCT02703714 & ॥ & 42 \\
\hline & Multicancer; advanced stage & PD-1 + bispecific CTLA-4/LAG3 & Pembrolizumab, XmAb 22841 & NCT03849469 & 1 & 242 \\
\hline & Multicancer; advanced stage & PD-1 + adoptive (mesothelin) & $\begin{array}{l}\text { Gavo-cel } \pm \text { fludarabine, } \\
\text { cyclophosphamide } \pm \text { anti-PD-1 }\end{array}$ & NCT03907852 & 1 & 70 \\
\hline \multirow[t]{3}{*}{ Other } & Multicancer; advanced stage & $\mathrm{IL}-27$ & SRF388 & NCT04374877 & I & 122 \\
\hline & Multicancer; advanced stage & Bispecific PD-L1/CD27 & CDX-527 & NCT04440943 & 1 & 96 \\
\hline & Multicancer; advanced stage & Mesothelin + JAK & LMB-100 immunotoxin, tofacitinib & NCT04034238 & 1 & 45 \\
\hline Adoptive & Advanced stage & Adoptive & TILs & NCT03801083 & $\|$ & 59 \\
\hline \multicolumn{7}{|l|}{$\mathrm{ICl}+$ targeted } \\
\hline \multirow[t]{5}{*}{ PARP } & $\begin{array}{l}\text { Advanced stage; } \\
\text { maintenance line }\end{array}$ & PARP + PD-1 & Rucaparib, nivolumab & NCT03639935 & ॥ & 32 \\
\hline & Multicancer; advanced stage & PARP + PD-1 & Olaparib, durvalumab & NCT03991832 & ॥ & 78 \\
\hline & Multicancer; advanced stage & PARP + PD-1 & Olaparib, pembrolizumab & NCT04123366 & $\|$ & 300 \\
\hline & Advanced stage & PARP + PD-1 & Olaparib, pembrolizumab & NCT04306367 & II & 29 \\
\hline & Multicancer; advanced stage & PARP + PD-1 + CTLA-4 & Olaparib, durvalumab, tremelimumab & NCT04169841 & ॥ & 270 \\
\hline Wnt & Advanced stage & Wnt/beta catenin & Nivolumab, DKN-01 & NCT04057365 & ॥ & 30 \\
\hline Multi-TKI & Multicancer; advanced stage & PD-1 + multi-TKI & Pembrolizumab, lenvatinib & NCT03797326 & ॥ & 760 \\
\hline HER2 & Multicancer; advanced stage & HER2 \pm PD-1 & SBT6050 \pm pembrolizumab & NCT04460456 & 1 & 294 \\
\hline \multicolumn{7}{|l|}{$\mathrm{ICI}+$ chemotherapy } \\
\hline \multirow[t]{4}{*}{ First line } & Advanced stage & PD-L1 & Gemcitabine, cisplatin \pm durvalumab & NCT03875235 & III & 757 \\
\hline & Advanced stage & PD-1 & Gemcitabine, cisplatin \pm pembrolizumab & NCT04003636 & III & 1,048 \\
\hline & Advanced stage & PD-L1, CTLA-4 & $\begin{array}{l}\text { Multi-arm: } \\
\text { gemcitabine } \pm \text { cisplatin, } \\
\text { durvalumab } \pm \text { tremelimumab }\end{array}$ & NCT03473574 & ॥ & 128 \\
\hline & Advanced stage & PD-L1 & $\begin{array}{l}\text { Gemcitabine, cisplatin, } \\
\text { atezolizumab } \pm \text { bevacizumab }\end{array}$ & NCT04677504 & ॥ & 162 \\
\hline Second line & Advanced stage & PD-1 & $\begin{array}{l}\text { Nivolumab, nano-liposomal irinotecan, } \\
\text { 5-FU }\end{array}$ & NCT03785873 & 1/II & 30 \\
\hline Nth line & Multicancer; advanced stage & PD-1 or CTLA-4 & $\begin{array}{l}\text { Intratumoral INT } 230-6 \pm \\
\text { pembrolizumab or ipilimumab }\end{array}$ & NCT03058289 & $1 / I I$ & 180 \\
\hline \multicolumn{7}{|l|}{$\mathrm{ICl}+\mathrm{LRT}$} \\
\hline Multiple & TACE or ablation & PD-1 + CTLA-4 & $\begin{array}{l}\text { Durvalumab + tremelimumab } \pm \\
\text { TACE/ablation }\end{array}$ & NCT02821754 & II & 90 \\
\hline \multirow[t]{3}{*}{ Radiation } & Hypofractionated & PD-1 \pm CTLA-4 & Nivolumab, radiation \pm ipilimumab & NCT02866383 & II & 160 \\
\hline & Hypofractionated & PD-1 + CTLA-4 & Durvalumab, tremelimumab, radiation & NCT03482102 & $\|$ & 70 \\
\hline & SIRT & PD-1 \pm CTLA- 4 & Durvalumab \pm tremelimumab, SIRT & NCT04238637 & ॥ & 50 \\
\hline
\end{tabular}

Abbreviations: BTC, biliary tract cancer; GM-CSF, granulocyte-macrophage colony-stimulating factor; ICl, immune checkpoint inhibitor; IL-27, interleukin-27; IO, immunotherapy; JAK, Janus kinase inhibitor; LRT, locoregional therapy; SIRT, selective internal radiotherapy; TACE, transarterial chemoembolization; TGF- $\beta$, transforming growth factor $\beta$; TILs, tumor-infiltrating lymphocytes; TKI, tyrosine kinase inhibitor.

TGF- $\beta$ Pathway

The bispecific therapeutic antibody bintrafusp alfa, cotargeting the TME modulator TGF- $\beta$ receptor, and PD- L1 showed clinical activity in a phase I trial involving patients with progressive BTC with an ORR of $20 \%{ }^{83}$ A subsequent phase II study of bintrafusp alfa as a second-line therapy in advanced BTC (ClinicalTrials.gov identifier: NCT03833661) failed to meet the primary endpoint, with an ORR of 10 . $1 \%{ }^{84}$ The first-line phase II/III trial of bintrafusp alfa with gemcitabine and cisplatin in patients with advanced BTC was recently closed based on an interim analysis (NCT04066491). Bintrafusp alfa in previously treated patients with advanced HCC $(\mathrm{n}=9)$ reported stable disease in 1 patient. $^{85}$ 


\section{Wnt Pathway}

In mouse and rat models of CCA, molecular crosstalk between TAMs and CCA cells was found to be mediated through the Wnt pathway, wherein Wnt inhibitors led to tumor regression. ${ }^{86}$ Furthermore, in a mouse model of HCC, tumors with beta catenin activation were associated with a loss of antigen-specific $\mathrm{CD}^{+} \mathrm{T}$ cells and $\mathrm{CD}_{103^{+}}$dendritic cells in TME, resulting in resistance to anti-PD-1 treatment. ${ }^{87}$ A monoclonal antibody targeting Dickkopf-1, a modulator of the Wnt pathway, used in a phase I study in combination with gemcitabine and cisplatin in patients with unresectable or metastatic BTC, was well tolerated (ClinicalTrials.gov identifier: NCT02375880), ${ }^{88}$ supporting a phase II trial of DKN-01 combined with nivolumab in patients with advanced refractory BTC (NCT04057365).

\section{MAPK Pathway}

Potentially actionable crosstalk between MAPK and tumor immune pathways has been noted, where MEK inhibitors were found to protect cytotoxic $\mathrm{T}$ cells from undergoing apoptosis in murine models. ${ }^{89}$ MAPK pathways are the downstream mediators of RAS/RTK signaling that defines the primary driver aberration in a significant subset of BTCs. MAPK inhibitors potentially represent a safe and at least partially effective therapy in biliary cancers, ${ }^{90}$ thus providing a rationale to test a combination of ICI therapy with MEK inhibitors. A randomized phase II study of atezolizumab \pm the MEK inhibitor cobimetinib in previously treated patients with BTC $(n=86)$ noted a modest improvement in PFS. ${ }^{91}$

\section{DNA Damage Repair Pathway}

PARP inhibition may enhance immunosuppression through the upregulation of PD-L1 expression ${ }^{92}$ or may lead to increased neoantigen presentation via tumor cell death in patients with DNA damage repair mutations, such as BRCA1/2 and PALB2. Therefore, PARP inhibitors and ICI strategy have been tested in ovarian cancers ${ }^{93,94}$ and metastatic castration-resistant prostate cancer, ${ }^{95}$ with promising early results. BRCA1/2 mutations are also identified in approximately $5 \%$ of patients with BTC and therefore may provide a mechanistic rationale for treatment using PARP inhibitors in combination with ICI. Multiple trials are ongoing to evaluate this hypothesis (Table 1).

\section{Adoptive T-Cell Therapy}

Adoptive T-cell therapy is a broader term that includes activated TIL therapy, engineered T-cell receptor therapy, or CAR T-cell therapy. TILs from freshly resected tumor tissue can be expanded ex vivo and treated using anti-PD-1 or CTLA-4 antibodies, or an anti-glucocorticoidinduced TNF receptor (GITR; eg, TNFRSF18) agonist, ${ }^{96}$ to enhance T-cell proliferation and allow for autologous Tcell infusion in BTCs. An adjuvant trial in 36 patients with resected BTCs who underwent activated T-cell transfer along with a dendritic cell vaccine reported significant improvement in median PFS and OS at 18.3 and 31.9 months, respectively, compared with 7.7 and 17.4 months, respectively, in patients treated with surgery alone. ${ }^{97}$ Additional case reports and small trials with encouraging data have been reported in HBTCs, and many trials are ongoing ${ }^{98}$ (eg, ClinicalTrials.gov identifier: NCT03801083). In addition, clinical trials with CAR $\mathrm{T}$ cells targeting the tumor antigen mesothelin in pancreaticobiliary cancers are underway (NCT03941626 and NCT03907852), per evidence shown of a manageable safety profile in phase I studies. ${ }^{99}$

\section{Perspective and Outlook}

To effectively deploy the full potential of immunotherapy, a deeper understanding of the tumor-specific immune milieu and factors influencing dynamic changes during disease progression and in response to standard local or systemic therapies is desperately needed. Although current genomic studies driving precision oncology efforts have largely focused on the identification of therapeutically actionable mutations in cancer cells, the genetic drivers of the TME including the immune component remain relatively less understood. The recent publication by Bagaev et $\mathrm{al}^{100}$ analyzing the pan-cancer TME underscores the dynamics of tumor tissue lineage-associated interplay between genomic and microenvironment features that impinge on the response to immunotherapy. Single-cell sequencing applications will significantly im-prove the understanding of the cellular composition of TMEs at a high resolution, including the identification of specific subtypes of infiltrating immune cells. ${ }^{101}$ Furthermore, the spatial organization of immune cells within the TME, especially in the context of LRT and systemic therapies, will help better define the immune cell dynamics, the relative enrichment of different subtypes of ICI targets to these therapies, and the potential markers of response and resistance.

Working within the framework of current trials, a more rigorous and systematic collection of tumor tissue and stool and blood samples, along with the incorporation of noninvasive imaging and analysis of cell-free DNA/circulating markers and the microbiome could help define correlative markers of response and optimize therapy. The somewhat scattered picture of the immune milieu of HBTCs gleaned from the literature also underscores the need for a more systematic analysis, using large patient datasets encompassing the cellular and molecular heterogeneity of tumor types. Spatiotemporal characterization of the tumor-immune milieu and its dynamic interplay needs to be performed in the context of standard, 


\section{Table 2. Ongoing Clinical Trials With ICI Therapy in HCC}

\begin{tabular}{|c|c|c|c|c|c|c|}
\hline Combination & Trial Characteristics & Targets & Agents & $\begin{array}{l}\text { ClinicalTrials.gov } \\
\text { Identifier }\end{array}$ & Phase & $\mathbf{N}$ \\
\hline \multicolumn{7}{|l|}{ PD-1/PD-L1 \pm other IO } \\
\hline \multirow[t]{9}{*}{ Monotherapy } & Advanced stage & PD-1/PD-L1 & Anti-PD-1/PD-L1 & NCT03949231 & III & 200 \\
\hline & $\begin{array}{l}\text { Recurrent; prior liver } \\
\text { transplant }\end{array}$ & PD-1 & JS001 & NCT03966209 & I & 20 \\
\hline & $\begin{array}{l}\text { Recurrent; prior liver } \\
\text { transplant }\end{array}$ & PD-1 & Camrelizumab & NCT04564313 & I & 20 \\
\hline & Adjuvant & PD-1 & Pembrolizumab & NCT03867084 & III & 950 \\
\hline & Adjuvant & PD-1 & Anti-PD-1 & NCT03914352 & $\mathrm{N} / \mathrm{A}$ & 40 \\
\hline & $\begin{array}{l}\text { Multicancer; intratumoral/ } \\
\text { arterial }\end{array}$ & PD-1 & Pembrolizumab & NCT03755739 & II/III & 200 \\
\hline & Multicancer; HIV & PD-1 & Pembrolizumab & NCT02595866 & I & 60 \\
\hline & Multicancer; advanced stage & PD-1 & INCB099280 & NCT04242199 & 1 & 140 \\
\hline & Multiple; advanced stage & PD-1 & Toripalimab & NCT03474640 & I & 258 \\
\hline \multirow{10}{*}{ PD-1/PD-L1 + other } & Neoadjuvant & PD-1 + CTLA-4 & Nivolumab, ipilimumab & NCT03510871 & ॥ & 40 \\
\hline & Neoadjuvant & PD-1 + CTLA-4 & Nivolumab, ipilimimab & NCT03682276 & $\mathrm{I} / \mathrm{II}$ & 32 \\
\hline & Advanced stage & PD-1 + TIM-3 & TSR-042, TSR-022 & NCT03680508 & $\|$ & 42 \\
\hline & Neoadjuvant & PD-1 \pm LAG3 & Nivolumab, relatlimab & NCT04658147 & I & 20 \\
\hline & Multicancer; advanced stage & $\begin{array}{l}\text { IL-15 + PD-1/ } \\
\text { PD-L1 } \pm \text { NK cells }\end{array}$ & $\begin{array}{l}\text { N-803 + PD-1/PD-L1 } \pm \text { PD-L1 } \\
\text { t-haNK }\end{array}$ & NCT03228667 & $\|$ & 636 \\
\hline & Advanced stage & PD-1 + IL-2 & $\begin{array}{l}\text { Nivolumab, IRX-2, } \\
\text { cyclophosphamide }\end{array}$ & NCT03655002 & I & 28 \\
\hline & $\begin{array}{l}\text { Advanced stage; } \\
\text { fibrolamellar }\end{array}$ & PD-1 + IFN- $\alpha 2 B$ & $\begin{array}{l}\text { FU, nivolumab, recombinant } \\
\text { IFN- } \alpha 2 \text { b-like protein }\end{array}$ & NCT04380545 & $1 / I I$ & 15 \\
\hline & $\begin{array}{l}\text { Advanced stage; } \\
\text { fibrolamellar }\end{array}$ & DNAJB1-PRKACA & $\begin{array}{l}\text { Nivolumab, ipilimumab, } \\
\text { DNAJB1-PRKACA vaccine }\end{array}$ & NCT04248569 & I & 12 \\
\hline & Multicancer; advanced stage & PD-1 + vaccine & $\begin{array}{l}\text { DPX-survivac, cyclophosphamide, } \\
\text { pembrolizumab }\end{array}$ & NCT03836352 & II & 184 \\
\hline & Multicancer; advanced stage & Adoptive \pm PD-1 \pm IL-2 & $\begin{array}{l}\text { FT500, nivolumab/ } \\
\text { pembrolizumab/atezolizumab, } \\
\text { cyclophosphamide, fludarabine, } \\
\text { IL-2 }\end{array}$ & NCT03841110 & I & 76 \\
\hline \multirow[t]{2}{*}{ Other } & Multicancer; advanced stage & IL-27 & SRF388 & NCT04374877 & I & 122 \\
\hline & Multicancer; advanced stage & PRL3 & PRL3-zumab & NCT04118114 & II & 30 \\
\hline \multirow[t]{11}{*}{ Adoptive } & Localized stage; post-TACE & $\mathrm{T}$ cell & Immuncell-LC & NCT02856815 & ॥ & 78 \\
\hline & Multicancer; advanced stage & T cell & IMA202, TCR-engineered T cells & NCT03441100 & I & 15 \\
\hline & Multicancer; advanced stage & $T$ cell & Autologous $\gamma \delta \mathrm{T}$ cells & NCT04032392 & I & 20 \\
\hline & Advanced stage & T cell & CAR T cells (GPC3) & NCT04121273 & I & 20 \\
\hline & Any stage & $\mathrm{T}$ cell & Allogeneic $\gamma \delta \mathrm{T}$ cells & NCT04518774 & I & 8 \\
\hline & Any stage & T cell & $\begin{array}{l}\text { Cytoxan, fludarabine } \rightarrow \\
\text { CAR T cells (GPC3) }\end{array}$ & NCT02905188 & I & 14 \\
\hline & Multicancer; advanced stage & $\mathrm{T}$ cell & CAR T cells (GPC3 and/or TGF- $\beta$ ) & NCT03198546 & I & 30 \\
\hline & Multicancer; advanced stage & $\mathrm{T}$ cell & $\begin{array}{l}\text { Cytoxan, fludarabine } \rightarrow \\
\text { CAR T/TCR T cells } \\
\text { (several targets) }\end{array}$ & NCT03941626 & $\mathrm{I} / \mathrm{II}$ & 50 \\
\hline & Multicancer & T cell & CAR-T cells & NCT03638206 & $\mathrm{I} / \mathrm{II}$ & 73 \\
\hline & Advanced stage & T cells & Autologous TCR-mimic T cells & NCT04502082 & $\mathrm{I} / \mathrm{II}$ & 50 \\
\hline & Multicancer; advanced stage & T cell + NK cell & iNKT cells, CD8+ T cells & NCT03093688 & $\mathrm{I} / \mathrm{II}$ & 40 \\
\hline \multicolumn{7}{|l|}{$\mathrm{ICl}+$ targeted } \\
\hline \multirow[t]{3}{*}{ Multi-TKI } & Advanced stage & PD-1 + multi-TKI & Nivolumab, lenvatinib & NCT03841201 & II & 50 \\
\hline & Advanced stage & PD-1 + multi-TKI & Pembrolizumab, cabozantinib & NCT04442581 & ॥ & 29 \\
\hline & $\begin{array}{l}\text { Multicancer; advanced } \\
\text { stage; HIV }\end{array}$ & PD-1 + multi-TKI & Nivolumab, cabozantinib & NCT04514484 & 1 & 18 \\
\hline \multirow[t]{2}{*}{ VEGF } & Adjuvant & $P D-1+V E G F$ & Sintilimab, bevacizumab & NCT04682210 & III & 246 \\
\hline & Neoadjuvant & PD-1 + VEGF & Atezolizumab, bevacizumab & NCT04721132 & II & 30 \\
\hline MET & Multicancer; advanced stage & PD-1 + cMET1 & APL-501 or nivolumab, APL-101 & NCT03655613 & $\mathrm{I} / \mathrm{II}$ & 119 \\
\hline
\end{tabular}




\begin{tabular}{|c|c|c|c|c|c|c|}
\hline \multirow[t]{2}{*}{ Ablation } & Thermal ablation & PD-1 & Toripalimab \pm thermal ablation & NCT03864211 & I/II & 130 \\
\hline & Cryoablation & PD-1 + VEGF & $\begin{array}{l}\text { Camrelizumab, apatinib, } \\
\text { cryoablation }\end{array}$ & NCT04724226 & ॥ & 34 \\
\hline \multirow{3}{*}{ Radiation } & Hypofractionated & PD-L1 \pm CTLA-4 & $\begin{array}{l}\text { Radiation }+ \text { durvalumab } \pm \\
\text { tremelimumab }\end{array}$ & NCT04430452 & II & 30 \\
\hline & SBRT & PD-1 & SBRT \pm sintilimab & NCT04167293 & II/III & 116 \\
\hline & SBRT & PD-1 & Sintilimab \pm SBRT & NCT04547452 & ॥ & 84 \\
\hline \multirow[t]{6}{*}{ Embolization } & Conventional TACE & PD-1 + multi-TKI & TACE, PD-1, lenvatinib & NCT04273100 & ॥ & 56 \\
\hline & DEB-TACE & PD-1 or multi-TKI & TACE + sorafenib/lenvatinib/PD-1 & NCT04229355 & III & 90 \\
\hline & DEB-TACE & PD-L1 + CTLA-4 & $\begin{array}{l}\text { TACE } \rightarrow \text { durvalumab, } \\
\text { tremelimumab }\end{array}$ & NCT03638141 & II & 30 \\
\hline & TACE & Adoptive & $\mathrm{TACE} \pm \mathrm{IKC}$ & NCT03592706 & II/III & 60 \\
\hline & TACE (adjuvant) & PD-1 + VEGFR2 & $\begin{array}{l}\text { Carrelizumab, apatinib } \rightarrow \text { surgery } \rightarrow \\
\text { TACE } \rightarrow \text { carrelizumab, apatinib }\end{array}$ & NCT04521153 & $\mathrm{N} / \mathrm{A}$ & 514 \\
\hline & TACE or TAE & PD-1 & TACE/TAE \pm nivolumab & NCT04268888 & II/III & 522 \\
\hline \multirow[t]{2}{*}{ Multiple } & $\begin{array}{l}\text { DEB-TACE or brachytherapy } \\
\text { or ablation }\end{array}$ & PD-1 & $\begin{array}{l}\text { Pembrolizumab, RFA/MWA/ } \\
\text { brachytherapy/TACE }\end{array}$ & NCT03753659 & ॥ & 30 \\
\hline & $\begin{array}{l}\text { TACE } \pm \text { hypofractionated } \\
\text { RT }\end{array}$ & PD-1 & Sintilimab, TACE \pm radiation & NCT04653389 & ॥ & 30 \\
\hline
\end{tabular}

Abbreviations: DEB, doxorubicin eluting beads; HAl, hepatic artery infusion; IKC, immune killer cells; IL, interleukin; MWA, microwave ablation; RFA, radiofrequency ablation; SBRT, stereotactic body radiotherapy; SIRT, selective internal radiotherapy; TACE, transarterial chemoembolization; TAE, transarterial embolization; TCR, T-cell receptor; TGF- $\beta$, transforming growth factor- $\beta$; TKI, tyrosine kinase inhibitor; VEGF, vascular endothelial growth factor.

targeted immune therapies using more consistent definitions and methodologies. There is also a need to go beyond observational studies to more experimental approaches to help define precision therapeutics incorporating potent immunotherapies. Finally, the effect of other ICI antibodies against TIM3, CD73, TIGIT, and LAG3 needs to be elucidated in combination with other immunotherapies.

\section{Conclusions}

HBTC remains a deadly disease with an urgent need to find new and better targets and therapies. Immunotherapy is a promising avenue to pursue, especially considering its early success in HCC and other solid tumors. However, given the varied and complex immunosuppressive microenvironment, combinatorial and personalized approaches are necessary, but current limited knowledge, lack of preclinical models, and, to a certain degree, rigid clinical trial designs remain challenges to maximizing the potential of truly improving outcomes in this disease.

Submitted February 15, 2021; final revision received July 28, 2021; accepted for publication September 22, 2021.

Disclosures: Dr. Kumar-Sinha has disclosed not receiving any financial consideration from any person or organization to support the preparation analysis, results, or discussion of this article. Dr. Sahai has disclosed serving as a Principal Investigator for Agios, Bristol-Myers Squibb, Celgene, Clovis, Exelixis, Fibrogen, Incyte, Ipsen, Medlmmune, Merck, NCl, and Rafael; as a consultant and advisory board member for AstraZeneca, GlaxoSmithKline, Histosonics, Incyte, QED, and Rafael; and on the data safety monitoring board for Incyte.

Correspondence: Vaibhav Sahai, MBBS, MS, Division of Hematology and Oncology, Department of Internal Medicine, University of Michigan Rogel Cancer Center, 1500 East Medical Center Drive, Ann Arbor, MI 48109-

5948. Email: vsahai@umich.edu 


\section{References}

1. Nakamura H, Arai $Y$, Totoki $Y$, et al. Genomic spectra of biliary tract cancer. Nat Genet 2015:47:1003-1010.

2. Rebouissou S, Nault JC. Advances in molecular classification and precision oncology in hepatocellular carcinoma. J Hepatol 2020;72:215-229.

3. Dhanasekaran R, Nault JC, Roberts LR, et al. Genomic medicine and implications for hepatocellular carcinoma prevention and therapy. Gastroenterology 2019;156:492-509.

4. Forner A, Reig M, Bruix J. Hepatocellular carcinoma. Lancet 2018;391: 1301-1314.

5. Jusakul A, Cutcutache I, Yong $\mathrm{CH}$, et al. Whole-genome and epigenomic landscapes of etiologically distinct subtypes of cholangiocarcinoma. Cancer Discov 2017;7:1116-1135.

6. Lowery MA, Ptashkin R, Jordan E, et al. Comprehensive molecular profiling of intrahepatic and extrahepatic cholangiocarcinomas: potential targets for intervention. Clin Cancer Res 2018;24:4154-4161.

7. Zou S, Li J, Zhou H, et al. Mutational landscape of intrahepatic cholangiocarcinoma. Nat Commun 2014;5:5696.

8. Jiao Y, Pawlik TM, Anders RA, et al. Exome sequencing identifies frequent inactivating mutations in BAP1, ARID1A and PBRM1 in intrahepatic cholangiocarcinomas. Nat Genet 2013;45:1470-1473.

9. Narayan RR, Creasy JM, Goldman DA, et al. Regional differences in gallbladder cancer pathogenesis: insights from a multi-institutional comparison of tumor mutations. Cancer 2019;125:575-585.

10. Li M, Zhang Z, Li X, et al. Whole-exome and targeted gene sequencing of gallbladder carcinoma identifies recurrent mutations in the ErbB pathway. Nat Genet 2014;46:872-876.

11. Javle M, Lowery M, Shroff RT, et al. Phase II study of BGJ398 in patients with FGFR-altered advanced cholangiocarcinoma. J Clin Oncol 2018;36: 276-282.

12. Bahleda R, Italiano A, Hierro C, et al. Multicenter phase I study of erdafitinib (JNJ-42756493), oral pan-fibroblast growth factor receptor inhibitor, in patients with advanced or refractory solid tumors. Clin Cancer Res 2019;25:4888-4897.

13. Abou-Alfa GK, Sahai V, Hollebecque A, et al. Pemigatinib for previously treated, locally advanced or metastatic cholangiocarcinoma: a multicentre, open-label, phase 2 study. Lancet Oncol 2020;21:671-684.

14. Lavingia $V$, Fakih M. Impressive response to dual BRAF and MEK inhibition in patients with BRAF mutant intrahepatic cholangiocarcinoma-2 case reports and a brief review. J Gastrointest Oncol 2016;7:E98-102.

15. Bogenberger JM, DeLeon TT, Arora M, et al. Emerging role of precision medicine in biliary tract cancers. NPJ Precis Oncol 2018;2:21.

16. Pellino $A$, Loupakis $F$, Cadamuro $M$, et al. Precision medicine in cholangiocarcinoma. Transl Gastroenterol Hepatol 2018;3:40.

17. Gubin MM, Zhang X, Schuster H, et al. Checkpoint blockade cancer immunotherapy targets tumour-specific mutant antigens. Nature 2014; 515:577-581

18. Patel SJ, Sanjana NE, Kishton RJ, et al. Identification of essential genes for cancer immunotherapy. Nature 2017;548:537-542.

19. Meng $X$, Huang Z, Teng F, et al. Predictive biomarkers in PD-1/PD-L1 checkpoint blockade immunotherapy. Cancer Treat Rev 2015;41: 868-876

20. Łuksza M, Riaz N, Makarov V, et al. A neoantigen fitness model predicts tumour response to checkpoint blockade immunotherapy. Nature 2017; 551:517-520.

21. Cristescu R, Mogg R, Ayers M, et al. Pan-tumor genomic biomarkers for PD-1 checkpoint blockade-based immunotherapy. Science 2018;362: eaar3593.

22. Kelley RK, Sangro B, Harris WP, et al. Efficacy, tolerability, and biologic activity of a novel regimen of tremelimumab $(T)$ in combination with durvalumab (D) for patients (pts) with advanced hepatocellular carcinoma (aHCC) [abstract]. J Clin Oncol 2020;38(Suppl):Abstract 4508.

23. Finn RS, Ryoo BY, Merle P, et al. Pembrolizumab as second-line therapy in patients with advanced hepatocellular carcinoma in KEYNOTE-240: a randomized, double-blind, phase III trial. J Clin Oncol 2020;38:193-202.

24. Yau T, Kang YK, Kim TY, et al. Efficacy and safety of nivolumab plus ipilimumab in patients with advanced hepatocellular carcinoma previously treated with sorafenib: the CheckMate 040 randomized clinical trial. JAMA Oncol 2020;6:e204564.

25. Chen DS, Mellman I. Elements of cancer immunity and the cancerimmune set point. Nature 2017;541:321-330.
26. Bian J, Lin J, Long J, et al. T lymphocytes in hepatocellular carcinoma immune microenvironment: insights into human immunology and immunotherapy. Am J Cancer Res 2020;10:4585-4606.

27. Sia D, Jiao Y, Martinez-Quetglas I, et al. Identification of an immunespecific class of hepatocellular carcinoma, based on molecular features. Gastroenterology 2017;153:812-826.

28. Hopkins BD, Pauli C, Du X, et al. Suppression of insulin feedback enhances the efficacy of PI3K inhibitors. Nature 2018;560:499-503.

29. Foerster F, Hess M, Gerhold-Ay A, et al. The immune contexture of hepatocellular carcinoma predicts clinical outcome. Sci Rep 2018;8: 5351.

30. Pfister D, Núñez NG, Pinyol R, et al. NASH limits anti-tumour surveillance in immunotherapy-treated HCC. Nature 2021;592:450-456.

31. Bozward AG, Warricker F, Oo YH, et al. Natural killer cells and regulatory $T$ cells cross talk in hepatocellular carcinoma: exploring therapeutic options for the next decade. Front Immunol 2021;12:643310.

32. Guan X, Lu Y, Zhu H, et al. The crosstalk between cancer cells and neutrophils enhances hepatocellular carcinoma metastasis via neutrophil extracellular traps-associated cathepsin G component: a potential therapeutic target. J Hepatocell Carcinoma 2021;8:451-465.

33. Osei-Bordom D, Bozward AG, Oo YH. The hepatic microenvironment and regulatory T cells. Cell Immunol 2020;357:104195.

34. Ma C, Han M, Heinrich B, et al. Gut microbiome-mediated bile acid metabolism regulates liver cancer via NKT cells. Science 2018;360: eaan5931.

35. Rimassa L, Personeni N, Aghemo A, et al. The immune milieu of cholangiocarcinoma: from molecular pathogenesis to precision medicine. J Autoimmun 2019;100:17-26.

36. Tariq NU, Vogel A, McNamara MG, et al. Biliary tract cancer: implicated immune-mediated pathways and their associated potential targets. Oncol Res Treat 2018;41:298-304.

37. Banales JM, Cardinale V, Carpino G, et al. Expert consensus document: cholangiocarcinoma: current knowledge and future perspectives consensus statement from the European Network for the Study of Cholangiocarcinoma (ENS-CCA). Nat Rev Gastroenterol Hepatol 2016;13:261280.

38. Job S, Rapoud D, Dos Santos A, et al. Identification of four immune subtypes characterized by distinct composition and functions of tumo microenvironment in intrahepatic cholangiocarcinoma. Hepatology 2020;72:965-981.

39. Zhao $\mathrm{Q}$, Huang ZL, He M, et al. BTLA identifies dysfunctional PD-1expressing $\mathrm{CD4}^{+} \mathrm{T}$ cells in human hepatocellular carcinoma. Oncolmmunology 2016;5:e1254855.

40. Toubal A, Nel I, Lotersztajn S, et al. Mucosal-associated invariant T cells and disease. Nat Rev Immunol 2019;19:643-657.

41. Budhu A, Forgues M, Ye QH, et al. Prediction of venous metastases, recurrence, and prognosis in hepatocellular carcinoma based on a unique immune response signature of the liver microenvironment. Cancer Cell 2006;10:99-111.

42. Zhang JP, Yan J, Xu J, et al. Increased intratumoral IL-17-producing cells correlate with poor survival in hepatocellular carcinoma patients. J Hepatol 2009;50:980-989.

43. Kinoshita M, Kobayashi S, Gotoh K, et al. Heterogeneity of Treg/Th17 according to cancer progression and modification in biliary tract cancers via self-producing cytokines. Dig Dis Sci 2020;65:2937-2948.

44. Ikemoto T, Shimada M, Ishikawa D, et al. Peripheral Tr1 and Foxp3 Treg as markers of recurrent malignancies in patients with hepato-biliary pancreatic cancers. Anticancer Res 2017;37:5541-5552.

45. Gabrielson A, Wu Y, Wang H, et al. Intratumoral CD3 and CD8 T-cell densities associated with relapse-free survival in HCC. Cancer Immunol Res 2016;4:419-430.

46. Ma J, Zheng B, Goswami S, et al. PD $1^{\mathrm{Hi}} \mathrm{CD} 8^{+} \mathrm{T}$ cells correlate with exhausted signature and poor clinical outcome in hepatocellular carcinoma. J Immunother Cancer 2019;7:331.

47. Wang $Y$, Ding $M$, Zhang $Q$, et al. Activation or suppression of the immune response mediators in biliary tract cancer (BTC) patients: a systematic review and meta-analysis. J Cancer 2017;8:74-84.

48. Tanaka R, Kimura K, Eguchi S, et al. Preoperative neutrophil-to-lymphocyte ratio predicts tumor-infiltrating $C D 8^{+} \mathrm{T}$ cells in biliary tract cancer. Anticancer Res 2020;40:2881-2887.

49. Gani F, Nagarajan N, Kim Y, et al. Program death 1 immune checkpoint and tumor microenvironment: implications for patients with intrahepatic cholangiocarcinoma. Ann Surg Oncol 2016;23:2610-2617. 
50. Tian L, Ma J, Ma L, et al. PD-1/PD-L1 expression profiles within intrahepatic cholangiocarcinoma predict clinical outcome. World J Surg Oncol 2020;18:303.

51. Yu F, Gong L, Mo Z, et al. Programmed death ligand-1, tumor infiltrating lymphocytes and HLA expression in Chinese extrahepatic cholangiocarcinoma patients: possible immunotherapy implications. Biosci Trends 2019;13:58-69.

52. Ghidini M, Cascione $\mathrm{L}$, Carotenuto $\mathrm{P}$, et al. Characterisation of the immune-related transcriptome in resected biliary tract cancers. Eur J Cancer 2017;86:158-165.

53. DiNardo CD, Stein EM, de Botton $\mathrm{S}$, et al. Durable remissions with ivosidenib in IDH1-mutated relapsed or refractory AML. N Engl J Med 2018;378:2386-2398.

54. Umemoto K, Togashi $Y$, Arai $Y$, et al. The potential application of PD-1 blockade therapy for early-stage biliary tract cancer. Int Immunol 2020; 32:273-281.

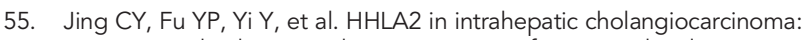
an immune checkpoint with prognostic significance and wider expression compared with PD-L1. J Immunother Cancer 2019;7:77.

56. Lu JC, Zeng HY, Sun QM, et al. Distinct PD-L1/PD1 profiles and clinical implications in intrahepatic cholangiocarcinoma patients with different risk factors. Theranostics 2019;9:4678-4687.

57. Pinato DJ, Guerra N, Fessas P, et al. Immune-based therapies for hepatocellular carcinoma. Oncogene 2020;39:3620-3637.

58. Kim RD, Chung V, Alese OB, et al. A phase 2 multi-institutional study of nivolumab for patients with advanced refractory biliary tract cancer. JAMA Oncol 2020;6:888-894.

59. Piha-Paul SA, Oh DY, Ueno M, et al. Efficacy and safety of pembrolizumab for the treatment of advanced biliary cancer: results from the KEYNOTE-158 and KEYNOTE-028 studies. Int J Cancer 2020;147: 2190-2198.

60. El-Khoueiry AB, Sangro B, Yau T, et al. Nivolumab in patients with advanced hepatocellular carcinoma (CheckMate 040): an open-label, non-comparative, phase $1 / 2$ dose escalation and expansion trial. Lancet 2017;389:2492-2502.

61. Zhu AX, Finn RS, Edeline J, et al. Pembrolizumab in patients with advanced hepatocellular carcinoma previously treated with sorafenib (KEYNOTE-224): a non-randomised, open-label phase 2 trial. Lancet Oncol 2018;19:940-952.

62. Benson AB III, D'Angelica MI, Abbott DE, et al. NCCN Clinical Practice Guidelines in Oncology: Hepatobiliary Cancers. Version 5.2021. Accessed December 28, 2021. To view the most recent version, visit NCCN.org

63. Yau T, Park JW, Finn RS, et al. CheckMate 459: a randomized, multicenter phase III study of nivolumab (NIVO) vs sorafenib (SOR) as firstline (1L) treatment in patients (pts) with advanced hepatocellular carcinoma (aHCC) [abstract]. Ann Oncol 2019;30(Suppl 5):Abstract LBA38_PR

64. Marabelle A, Le DT, Ascierto PA, et al. Efficacy of pembrolizumab in patients with noncolorectal high microsatellite instability/mismatch repair-deficient cancer: results from the phase II KEYNOTE-158 study. J Clin Oncol 2020;38:1-10.

65. loka T, Ueno M, Oh DY, et al. Evaluation of safety and tolerability of durvalumab (D) with or without tremelimumab (T) in patients (pts) with biliary tract cancer (BTC) [abstract]. J Clin Oncol 2019;37(Suppl):Abstract 387.

66. Parry RV, Chemnitz JM, Frauwirth KA, et al. CTLA-4 and PD-1 receptors inhibit T-cell activation by distinct mechanisms. Mol Cell Biol 2005;25: 9543-9553.

67. Klein $O$, Kee D, Nagrial A, et al. Evaluation of combination nivolumab and ipilimumab immunotherapy in patients with advanced biliary tract cancers: subgroup analysis of a phase 2 nonrandomized clinical trial. JAMA Oncol 2020;6:1405-1409.

68. Sahai V, Griffith KA, Beg MS, et al. A multicenter randomized phase II study of nivolumab in combination with gemcitabine/cisplatin or ipilimumab as first-line therapy for patients with advanced unresectable biliary tract cancer (BilT-01) [abstract]. J Clin Oncol 2020;38(Suppl):Abstract 4582.

69. Puhr HC, Ilhan-Mutlu A. New emerging targets in cancer immunotherapy: the role of LAG3. ESMO Open 2019;4:e000482.

70. Qin SK, Li Q, Ming Xu J, et al. Icaritin-induced immunomodulatory efficacy in advanced hepatitis B virus-related hepatocellular carcinoma: immunodynamic biomarkers and overall survival. Cancer Sci 2020;111: 4218-4231.
71. Sato $Y$, Tanaka S, Kinoshita M, et al. Immunosuppressive tumor microenvironment in occupational cholangiocarcinoma: supportive evidence for the efficacy of immune checkpoint inhibitor therapy. J Hepatobiliary Pancreat Sci 2020;27:860-869.

72. Xue Y, Gao S, Gou J, et al. Platinum-based chemotherapy in combination with PD-1/PD-L1 inhibitors: preclinical and clinical studies and mechanism of action. Expert Opin Drug Deliv 2021;18:187-203.

73. Oh DY, Lee KH, Lee DW, et al. Phase II study assessing tolerability, efficacy, and biomarkers for durvalumab (D) \pm tremelimumab (T) and gemcitabine/cisplatin (GemCis) in chemo-naïve advanced biliary tract cancer (aBTC) [abstract]. J Clin Oncol 2020;38(Suppl):Abstract 4520.

74. Imfinzi plus chemotherapy significantly improved overall survival in 1 stline advanced biliary tract cancer in TOPAZ-1 phase III trial at interim analysis [news release]. Cambridge, UK; October 5, 2021. Available at: https://www.astrazeneca.com/media-centre/press-releases/2021/imfinziimproved-survival-in-biliary-tract-cancer.html

75. de la Torre-Alaez M, Matilla A, Varela M, et al. Nivolumab after selective internal radiation therapy (SIRT) using SIR-spheres resin microspheres in patients with hepatocellular carcinoma: the NASIR-HCC trial [abstract]. Presented at the 2020 ILCA Virtual Conference; September 11-13, 2020. Abstract 0-27.

76. Xie C, Duffy AG, Mabry-Hrones D, et al. Tremelimumab in combination with microwave ablation in patients with refractory biliary tract cancer. Hepatology 2019;69:2048-2060.

77. Goodman AM, Kato S, Bazhenova L, et al. Tumor mutational burden as an independent predictor of response to immunotherapy in diverse cancers. Mol Cancer Ther 2017:16:2598-2608.

78. Li J, Wei Q, Wu X, et al. Integrative clinical and molecular analysis of advanced biliary tract cancers on immune checkpoint blockade reveals potential markers of response. Clin Transl Med 2020;10:e118.

79. Wallin JJ, Bendell JC, Funke R, et al. Atezolizumab in combination with bevacizumab enhances antigen-specific T-cell migration in metastatic renal cell carcinoma. Nat Commun 2016;7:12624.

80. Finn RS, Qin S, Ikeda M, et al. Atezolizumab plus bevacizumab in unresectable hepatocellular carcinoma. N Engl J Med 2020;382:1894-1905

81. Lin J, Yang $X$, Long J, et al. Pembrolizumab combined with lenvatinib as non-first-line therapy in patients with refractory biliary tract carcinoma. Hepatobiliary Surg Nutr 2020;9:414-424.

82. Rahma OE, Hodi FS. The intersection between tumor angiogenesis and immune suppression. Clin Cancer Res 2019;25:5449-5457.

83. Yoo C, Oh DY, Choi HJ, et al. Phase I study of bintrafusp alfa, a bifunctional fusion protein targeting TGF- $\beta$ and PD-L1, in patients with pretreated biliary tract cancer. J Immunother Cancer 2020;8:e000564.

84. Merck KGaA, Darmstadt, Germany, reports Topline data for bintrafusp alfa as second-line monotherapy treatment in biliary tract cancer. Accessed July 1, 2021. Available at: https://www.emdgroup.com/en/ news/bintrafusp-topline-data-biliary-tract-cancer-16-03-2021.html

85. Doi T, Fujiwara $Y$, Koyama $T$, et al. Phase I study of the bifunctional fusion protein bintrafusp alfa in Asian patients with advanced solid tumors, including a hepatocellular carcinoma safety-assessment cohort. Oncologist 2020;25:e1292-e1302.

86. Boulter L, Guest RV, Kendall TJ, et al. WNT signaling drives cholangiocarcinoma growth and can be pharmacologically inhibited. J Clin Invest 2015:125:1269-1285.

87. Ruiz de Galarreta M, Bresnahan E, Molina-Sánchez $P$, et al. $\beta$-catenin activation promotes immune escape and resistance to anti-PD-1 therapy in hepatocellular carcinoma. Cancer Discov 2019;9:1124-1141.

88. Goyal L, Sirard C, Schrag M, et al. Phase I and biomarker study of the Wnt pathway modulator DKN-01 in combination with gemcitabine/cisplatin in advanced biliary tract cancer. Clin Cancer Res 2020;26:6158-6167.

89. Ebert PJR, Cheung J, Yang Y, et al. MAP kinase inhibition promotes $T$ cell and anti-tumor activity in combination with PD-L1 checkpoint blockade. Immunity 2016;44:609-621.

90. Bekaii-Saab T, Phelps MA, Li X, et al. Multi-institutional phase II study of selumetinib in patients with metastatic biliary cancers. J Clin Oncol 2011:29:2357-2363.

91. Yarchoan M, Cope L, Anders RA, et al. A multicenter randomized phase 2 trial of atezolizumab as monotherapy or in combination with cobimetinib in biliary tract cancers (BTCs): a NCl Experimental Therapeutics Clinical Trials Network (ETCTN) study [abstract]. Cancer Res 2020;80 (26 Suppl):Abstract CT043

92. Jiao S, Xia W, Yamaguchi H, et al. PARP inhibitor upregulates PD-L1 expression and enhances cancer-associated immunosuppression. Clin Cancer Res 2017;23:3711-3720. 
93. Konstantinopoulos PA, Waggoner S, Vidal GA, et al. Single-arm phases 1 and 2 trial of niraparib in combination with pembrolizumab in patients with recurrent platinum-resistant ovarian carcinoma. JAMA Oncol 2019:5:1141-1149.

94. Higuchi T, Flies DB, Marjon NA, et al. CTLA-4 blockade synergizes therapeutically with PARP inhibition in BRCA1-deficient ovarian cancer. Cancer Immunol Res 2015;3:1257-1268.

95. Karzai F, VanderWeele D, Madan RA, et al. Activity of durvalumab plus olaparib in metastatic castration-resistant prostate cancer in men with and without DNA damage repair mutations. J Immunother Cancer 2018 ; 6:141.

96. Zhou G, Sprengers D, Mancham S, et al. Reduction of immunosuppressive tumor microenvironment in cholangiocarcinoma by ex vivo targeting immune checkpoint molecules. J Hepatol 2019;71: 753-762.
97. Shimizu K, Kotera Y, Aruga A, et al. Clinical utilization of postoperative dendritic cell vaccine plus activated T-cell transfer in patients with intrahepatic cholangiocarcinoma. J Hepatobiliary Pancreat Sci 2012;19:171-178.

98. Shi D, Shi Y, Kaseb AO, et al. Chimeric antigen receptor-glypican-3 Tcell therapy for advanced hepatocellular carcinoma: results of phase I trials. Clin Cancer Res 2020;26:3979-3989.

99. Anwar MY, Williams GR, Paluri RK. CAR T cell therapy in pancreaticobiliary cancers: a focused review of clinical data. J Gastrointest Cancer 2021;52:1-10

100. Bagaev A, Kotlov N, Nomie K, et al. Conserved pan-cancer microenvironment subtypes predict response to immunotherapy. Cancer Cell 2021;39:845-865.e7.

101. Gohil SH, lorgulescu JB, Braun DA, et al. Applying high-dimensional single-cell technologies to the analysis of cancer immunotherapy. Nat Rev Clin Oncol 2020;18:244-256. 